\title{
A metaheuristic approach to manufacturing process planning in reconfigurable manufacturing systems
}

\begin{abstract}
Manufacturing process planning (MPP) is concerned with decisions regarding selection of an optimal configuration for processing parts. For multiparts reconfigurable manufacturing lines, such decisions are strongly influenced by the types of processes available, the relationships for sequencing the processes and the order of processing parts. Decisions may conflict, hence the decision making tasks must be carried out in a concurrent manner. This paper outlines an optimization solution technique for the MPP problem in reconfigurable manufacturing systems (RMSs). MPP is modelled in an optimization perspective and the solution methodology is provided through a metaheuristic technique known as simulated annealing. Analytical functions for modelling MPP are based on knowledge of processes available to the manufacturing system as well as processing constraints. Application of this approach is illustrated through a multistage parallel-serial reconfigurable manufacturing line. The results show that significant improvements to the solution of this type of problem can be gained through the use of simulated annealing. Moreover, the metaheuristic technique is able to identify an optimal manufacturing process plan for a given production scenario.
\end{abstract}

Keyword: Metaheuristics; Simulated annealing; Manufacturing process planning;

Reconfigurable manufacturing systems; Production scenarios 